Relations industrielles

Industrial Relations

\title{
Milan Kubr et Joseph Prokopenko. Les besoins de formation en management: principes et méthodes de diagnostic
}

\section{Denis Morin}

Volume 47, numéro 2, 1992

URI : https://id.erudit.org/iderudit/050783ar

DOI : https://doi.org/10.7202/050783ar

Aller au sommaire du numéro

Éditeur(s)

Département des relations industrielles de l'Université Laval

ISSN

0034-379X (imprimé)

1703-8138 (numérique)

Découvrir la revue

Citer ce compte rendu

Morin, D. (1992). Compte rendu de [Milan Kubr et Joseph Prokopenko. Les besoins de formation en management: principes et méthodes de diagnostic]. Relations industrielles / Industrial Relations, 47(2), 388-390.

https://doi.org/10.7202/050783ar

Tous droits réservés @ C Département des relations industrielles de l'Université Laval, 1992
Ce document est protégé par la loi sur le droit d'auteur. L'utilisation des services d'Érudit (y compris la reproduction) est assujettie à sa politique d'utilisation que vous pouvez consulter en ligne.

https://apropos.erudit.org/fr/usagers/politique-dutilisation/ 
des ressources humaines? Une telle vision irait à l'encontre de la réalité puisque plusieurs études de cas ont montré l'interaction entre la planification stratégique et la planification des ressources humaines (Dyer 1984). Un autre aspect passé sous silence concerne les rôles que les professionnels de ressources humaines sont appelés à jouer dans ce processus de planification. La deuxième question de fond a trait à la vision très utilitariste adoptée par les auteurs pour intégrer les ressources humaines à la planification stratégique. Les auteurs insistent à plusieurs reprises sur l'importance des profits, de l'approche coût/bénéfice et de l'utilité. En filigrane le message de ce livre est le suivant: les intérêts des employés doivent passer après les impératifs économiques. Par exemple, à la page 2, on peut lire: The human resource department's responsibility should be defined as the maximisation of corporate profits through the better management and use of people. Ne vaudrait-il pas mieux parler de productivité que de simple maximisation des profits? Dans le même ordre d'idée, les auteurs émettent indirectement des réserves sur la compétence des professionnels de ressources humaines en écrivant: Human resource executives should be experienced business people (page 3). Le danger de cette prescription est de ne tenir compte que des intérêts des actionnaires (profits) au détriment de ceux des employés. Selon nous, ce n'est pas en mettant en sourdine les considérations sociales que les organisations vont réussir à redéfinir les relations d'emploi axées sur une plus grande coopération tant prônée pour surmonter le défi de la compétitivité.

Malgré ce biais, le livre de Lengnick-Hall et Lengnick-Hall vaut la peine d'être lu. Il aidera les étudiants et praticiens en relations industrielles à réaliser l'urgence de renforcer leurs compétences en gestion stratégique. Quant aux autres lecteurs, ils trouveront dans ce livre une synthèse originale des écrits en gestion stratégique des ressources humaines.

Thierry WILS

Université du Québec à Hull

Les besoins de formation en management: principes et méthodes de diagnostic, Série Formation à la gestion, $n^{\circ} 27$, par Milan Kubr et Joseph Prokopenko, Genève, Bureau international du travail, 1991, 317 p., ISBN 92-2-206399-6

L'évaluation des besoins de formation constitue le point de départ logique au développement et à l'évaluation des programmes de formation. L'évaluation rigoureuse des besoins de formation fournit des informations sur le niveau de formation nécessaire, la nature du contenu de la formation et les groupes d'employés susceptibles de recevoir de la formation.

Les recherches publiées sur le sujet se concentrent à priori sur le développement de nouvelles techniques et méthodes pour accroitre la qualité de l'information à recueillir lors de l'évaluation des besoins de formation. L'ouvrage de Kubr et Prokopenko s'avère essentiellement un guide méthodologique qui décrit la démarche à suivre pour effectuer un diagnostic complet et articulé des besoins de formation au niveau du management. Le livre dresse également l'inventaire des instruments en usage. Les auteurs portent une attention 
particulière à l'application de ces techniques tout en soulignant leurs qualités et limites. Le volume incorpore une perspective macroscopique (organisationnelle) et microscopique (groupe, individuelle) dans la discussion des méthodes associées à l'évaluation des besoins de formation.

L'ouvrage est divisé en dix chapitres formant trois parties. Dans la première section composée de deux chapitres, les auteurs résument quelques principes théoriques à la base de l'évaluation des besoins de formation. Kubr et Prokopenko établissent d'abord une distinction entre les interventions qui relèvent ou non de la formation. Ces auteurs soutiennent avec pertinence qu'il y a des situations où le rendement des cadres ne peut être amélioré par un programme de formation. Un programme de formation vise principalement à susciter un changement au niveau des habiletés, des comportements, des connaissances et des attitudes. La formation ne solutionnera pas les problèmes administratifs. Le processus de diagnostic permettra à cet effet de vérifier si les problèmes de rendement des cadres peuvent être abordés par le truchement d'activités de formation. Kubr et Prokopenko définissent un ensemble d'interventions pouvant augmenter le rendement des cadres, comme, par exemple, le développement organisationnel, la formation au management, le développement des cadres ou l'amélioration des pratiques organisationnelles. Ils nuancent les liens entre ces interventions et l'évaluation des besoins de formation. Un modèle d'identification des besoins de formation est également proposé. Ce cadre conceptuel illustre globalement la démarche d'analyse d'un problème de rendement ou de compétence. La détermination des besoins de formation exige un examen entre l'écart qui subsiste entre le niveau visé et le niveau actuel de performance. A partir de ce modèle, les auteurs exposent progressivement la démarche d'évaluation des besoins de formation.

La deuxième partie du volume traite de la valeur des différentes méthodes et instruments de diagnostic. Cette seconde section de l'ouvrage est remarquable par sa systématisation. Le troisième chapitre se concentre au préalable sur les procédures d'identification et d'analyse des problèmes. Cette étape constitue une phase essentielle de l'évaluation des besoins de formation. La présentation, l'analyse ainsi qu'une description des points forts et faibles caractérisant les différentes méthodes de diagnostic marquent le contenu de trois chapitres. Les instruments et les méthodes sont exposés avec précision. Les méthodes ont été subdivisées en trois grandes catégories selon qu'elles s'appliquent à l'évaluation des besoins individuels (chapitre 4), de groupes (chapitre 5) ou organisationnels (chapitre 6). La classification des divers instruments s'inspire, dans une certaine mesure, du cadre théorique de McGehee et Thayer. En effet, ce cadre théorique identifie trois niveaux d'analyse interreliés à considérer dans l'évaluation des besoins de formation: l'analyse organisationnelle, l'analyse des opérations (des tâches) et l'analyse individuelle. Kubr et Prokopenko ajoute l'analyse des processus internes du groupe au schéma d'analyse des besoins à trois dimensions. Les auteurs évoquent à cet égard certaines techniques de diagnostic telles que la réunion d'équipe, les sociogrammes et la formation par simulation (ex.: jeux d'entreprise, jeux dramatiques). Les écrits sur la formation ont longtemps négligé l'analyse des groupes pour se concentrer sur l'analyse individuelie. Par ailleurs, l'analyse des différentes méthodes d'évaluation des besoins de formation met en évidence que les mesures d'autodiagnostic (self-report measures) telles que les 
questionnaires et les entrevues sont les plus fréquemment utilisées. La détermination de la fidélité et de la validité de ces instruments doit constituer une préoccupation constante du formateur.

Dans la troisième partie du volume, les auteurs s'intéressent aux pratiques courantes en matière de diagnostic des besoins de formation. Le chapitre 7 consiste en un exposé sur l'utilisation et l'application des méthodes dans le milieu organisationnel. Depuis le début des années 80 , la tendance en regard de l'analyse organisationnelle est l'identification des besoins de formation en fonction de la stratégie corporative. Cette nouvelle approche postule que la planification stratégique doit être une partie intégrante des responsabilités du formateur afin de déterminer les besoins futurs de formation de la force de travail. Dans la même veine, la nouvelle tendance s'apparente à l'analyse des tâches qui se focalise sur ce qui sera requis en termes de comportements et aptitudes dans les emplois futurs. Les auteurs discutent largement de la planification des carrières et de ses ramifications avec l'identification des besoins de formation. La gestion de l'obsolescence des aptitudes au niveau du management constituera également un défi à relever dans l'identification des besoins de formation. Les auteurs abordent ainsi une conception stratégique de l'évaluation des besoins de formation à l'intérieur du chapitre 7 .

Les chapitres suivants se rapportent à des cas plus particuliers d'évaluation des besoins de formation. Le chapitre 8 aborde les enquêtes portant sur les besoins de formation à l'échelle sectorielle, nationale ou internationale. Le chapitre 9 est consacré aux institutions de développement des cadres confrontés à des problèmes lors de l'évaluation des besoins de formation. Enfin, le chapitre 10 décrit plus spécifiquement le diagnostic des besoins de formation dans les petites entreprises.

Le lecteur profitera avantageusement des annexes dans l'application des méthodes d'évaluation des besoins de formation. Une méthode fréquemment utilisée pour déterminer les besoins de formation est l'évaluation du rendement. L'annexe I présente une analyse détaillée des multiples techniques de notation en fonction de deux catégories: les techniques comparatives et les techniques absolues. L'annexe $I I$ fournit une analyse comparative des méthodes d'évaluation des besoins de formation selon le rapport coût-efficacité, la nature des besoins de formation et le cycle d'évaluation des besoins.

En conclusion, cet ouvrage sur les besoins de formation en management constitue un outil de référence fort intéressant. II convient parfaitement aux gestionnaires en ressources humaines ainsi qu'aux étudiants intéressés à l'étude des activités de formation. Par ailleurs, le volume est abondamment illustré de tableaux-synthèses. La présentation de plusieurs cas permet de nous familiariser sur la façon dont le diagnostic des besoins de formation s'effectue dans certaines organisations et dans plusieurs pays.

Denis Morin

Université Laval 